A. Y. T. Wu • N. C. T. Kong • F. A. de Leon · C. Y. Pan •

T. Y. Tai $\cdot$ V. T. F. Yeung $\cdot$ S. J. Yoo $\cdot$ A. Rouillon $\cdot$

M. R. Weir · for the MAPS Investigators

\title{
An alarmingly high prevalence of diabetic nephropathy in Asian type 2 diabetic patients: the MicroAlbuminuria Prevalence (MAP) Study
}

Received: 6 July 2004 / Accepted: 22 October 2004 / Published online: 23 December 2004

(C) Springer-Verlag 2004

\begin{abstract}
Aim/hypothesis: Microalbuminuria represents the earliest clinical evidence of diabetic nephropathy and is a marker of increased cardiovascular morbidity and

A.Y.T. Wu, F.A. de Leon and M.R. Weir received honoraria for speaking engagements and A. Rouillon is employed by SanofiSynthelabo Groupe.
\end{abstract}

A. Y. T. Wu $(\bowtie)$

Wu Nephrology and Medical Clinic, Mount Elizabeth Medical

Center,

3 Mount Elizabeth,

2258510, Singapore

e-mail: akirawu@pacific.net.sg

Tel.: +65-6-7321819

Fax: +65-6-7348266

N. C. T. Kong

Hospital University Kebangsaan Malaysia,

Kuala Lumpur, Malaysia

F. A. de Leon

Santo Tomas University Hospital,

Manila, Philippines

C. Y. Pan

Military General Hospital,

Beijing, People's Republic of China

T. Y. Tai

National Health Research Institute,

Taipei, Taiwan

V. T. F. Yeung

Our Lady of Maryknoll Hospital,

Hong Kong, China

S. J. Yoo

Catholic University of Korea Holy Family Hospital,

Seoul, South Korea

\section{A. Rouillon}

Sanofi-Synthelabo Groupe,

Gentilly, France

M. R. Weir

Division of Nephrology, University of Maryland School of Medicine,

Baltimore, MD, USA mortality. Its early detection allows the implementation of individualised and aggressive intervention programmes to reduce cardiovascular risk factors. There is limited information on the prevalence of microalbuminuria among hypertensive type 2 diabetic patients in Asia. Methods: This cross-sectional epidemiological study aimed to assess the prevalence of microalbuminuria and macroalbuminuria among consecutively screened hypertensive type 2 diabetic adult patients in 103 centres in 10 Asian countries or regions. Predictive factors for microalbuminuria and macroalbuminuria were characterised using a stepwise logistic regression model. Results: A total of 6,801 patients were enrolled and 5,549 patients constituted the per-protocol population (patients with bacteriuria and haematuria were excluded). The prevalence of microalbuminuria was $39.8 \%(39.2-40.5 ; 95 \% \mathrm{CI})$ and the prevalence of macroalbuminuria was 18.8\% (18.2-19.3; 95\% CI). Only $11.6 \%$ of the patients had systolic and diastolic blood pressure below the $130 / 80 \mathrm{~mm} \mathrm{Hg}$ target. In the multivariate analyses, the predictive factors for the presence of microalbuminuria were age, BMI, systolic blood pressure and ethnic origin. The highlighted predictive factors for the presence of macroalbuminuria were age, sex, ethnic origin, BMI, duration of diabetes, presence of diabetic complications, intake of diuretics, intake of calcium channel blockers, diastolic and systolic blood pressure. Conclusions/interpretation: The high prevalence $(58.6 \%)$ of micro or macroalbuminuria observed in these patients is alarming and indicates an impending pandemic of diabetic cardiovascular and renal diseases in Asia with its potential economic consequences.

Keywords Asia - Diabetic nephropathy - Hypertension · Macroalbuminuria - Microalbuminuria - Prevalence

Abbreviations ADA: American Diabetes Association ARB: angiotensin receptor blocker - MAPS: MicroAlbuminuria Prevalence Study - MAU: microalbuminuria 


\section{Introduction}

Hypertension is common among patients with type 2 diabetes, with a prevalence approximately twice that of the nondiabetic population, and may precede the onset of diabetes [1]. The prevalence of hypertension is further increased in patients with type 2 diabetes mellitus and elevated AER, compared with patients with type 2 diabetes mellitus and no evidence of renal involvement. The higher the systolic blood pressure, the greater the absolute excess cardiovascular risk for diabetic patients, indicating a greater potential for preventing cardiovascular death by control of elevated blood pressure [2]. Ageing of the population and an increasing prevalence of obesity and sedentary life habits are leading to an greater prevalence of diabetes, particularly in Asia [3]. By 2025, the World Health Organization predicts that the number of patients with the disease worldwide will increase to 300 million. Half will be Asian. Southeast Asia will have the highest rate of diabetes worldwide [4].

Because of the adverse impact of microalbuminuria (MAU) on survival in patients with type 2 diabetes mellitus and the renal risk of macroalbuminuria [5-8], screening and intervention programmes should be implemented early, at the stage of microalbuminuria. Annual screening for microalbuminuria is recommended by the American Diabetes Association (ADA) [9], as a high proportion of patients with type 2 diabetes are found to have MAU or overt nephropathy shortly after diagnosis of their diabetes. Screening by means of a semiquantitative dipstick test is easy, immediate and accurate [10].

To date there have been few studies in Asian populations on the prevalence of MAU [11-14]. These studies have only explored the percentage of MAU in diabetic or hypertensive patients. The MicroAlbuminuria Prevalence Study (MAPS) is the first large study to evaluate the prevalence of micro and macroalbuminuria in high-risk patients with type 2 diabetes mellitus and hypertension.

\section{Subjects and methods}

Patients and study design Outpatients who were above the age of 18, from different Asian ethnic subgroups, and had previously diagnosed hypertension (treated or untreated) and type 2 diabetes mellitus (treated or untreated) were consecutively screened at each participating centre. Previously diagnosed hypertension and diabetes were historically defined as mentioned in the patient medical record and verified during monitoring visits. The only exclusion criterion was previously known macroalbuminuria. Patient data included ethnic origin; past medical and family history; onset dates of hypertension and diabetes; current diabetes status with known complications such as retinopathy, peripheral neuropathy, cardiovascular disease as reported during the interview, as well as glycaemic control (latest values of fasting blood glucose and $\mathrm{HbA}_{1} \mathrm{c}$ if available); current hypertensive status (mean of two consecutive measurements of office supine systolic and diastolic blood pressure) and current therapy; and dyslipidaemic status (known or previously diagnosed dyslipidaemia), with latest blood tests results if available, and use of lipidlowering agents. A single urine specimen was collected in disposable plastic vessels.

The primary study objective was to assess the prevalence of microalbuminuria and macroalbuminuria. Secondary objectives aimed to assess the routine blood pressure control level, to collect the associated cardiovascular risk factors and diabetic complications, and to characterise the predictive factors for micro and macroalbuminuria.

Informed consent was obtained from all participants. The protocol was approved by the Ethics Committees in countries where it was required by the regulatory bodies. The study was supervised by a multidisciplinary steering committee. The committee designed the study, interpreted the data and wrote the article.

Assays A two-step MAU screening process was conducted. First, the detection of macroalbuminuria was carried out by trained personnel on fresh urine (first morning or random morning specimens) using a visual colorimetric semiquantitative urine test strip (Nephur 7 Test, Roche Diagnostics, Mannheim, Germany). The test strip also allowed quantification of $\mathrm{pH}$, urine glucose, ketones bodies, leucocytes, nitrites, and blood. If specimens were negative for albumin, detection of microalbuminuria was performed on the same urine with a second specific semiquantitative urine test strip (Micral-test, Roche Diagnostics). The intensity of the colour produced was visually compared with the reference chart on the Micral-test bottle $(0,20,50,100 \mathrm{mg} / \mathrm{l},>100$ $\mathrm{mg} / \mathrm{l})$. A measurement of $20 \mathrm{mg} / \mathrm{l}$ or above was considered positive. Specificity, sensitivity, positive and negative predictive values of the second test strip were determined according to the manufacturer's evaluation report and with a cutoff point set at $20 \mathrm{mg} / \mathrm{l}$. Results were: sensitivity $90.1 \%$, specificity $87.2 \%$, positive predictive value 0.82 , negative predictive value 0.93 .

Populations Between January 2002 and December 2002 we recruited 6,801 patients from 103 medical centres (30 general practitioner and primary care clinics, 19 general hospital outpatient clinics, 40 diabetic centres, and 14 cardiology or nephrology centres) throughout ten Asian countries or regions (China, Hong Kong, Indonesia, Malaysia, Pakistan, Philippines, Singapore, South Korea, Taiwan and Thailand).

The analysed population $(n=6,482)$ consisted of all patients with confirmed onset dates for hypertension and type 2 diabetes mellitus. Most patients $(n=3,070,47 \%)$ were enrolled by diabetologists, whereas $1,429(22 \%)$, $1,236(19 \%)$ and 747 patients $(12 \%)$ were enrolled by general practitioners, hospital outpatient clinic physicians and specialists (cardiologists or nephrologists), respectively. Patients with positive leucocytes and nitrites, which are indicative of significant bacteriuria, and patients with erythrocytes or haemoglobin equal to or above 25 counts per microlitre, which is indicative of significant haematuria (false positives), were excluded from the analysed 
Fig. 1 Patients' disposition and urinary screening results

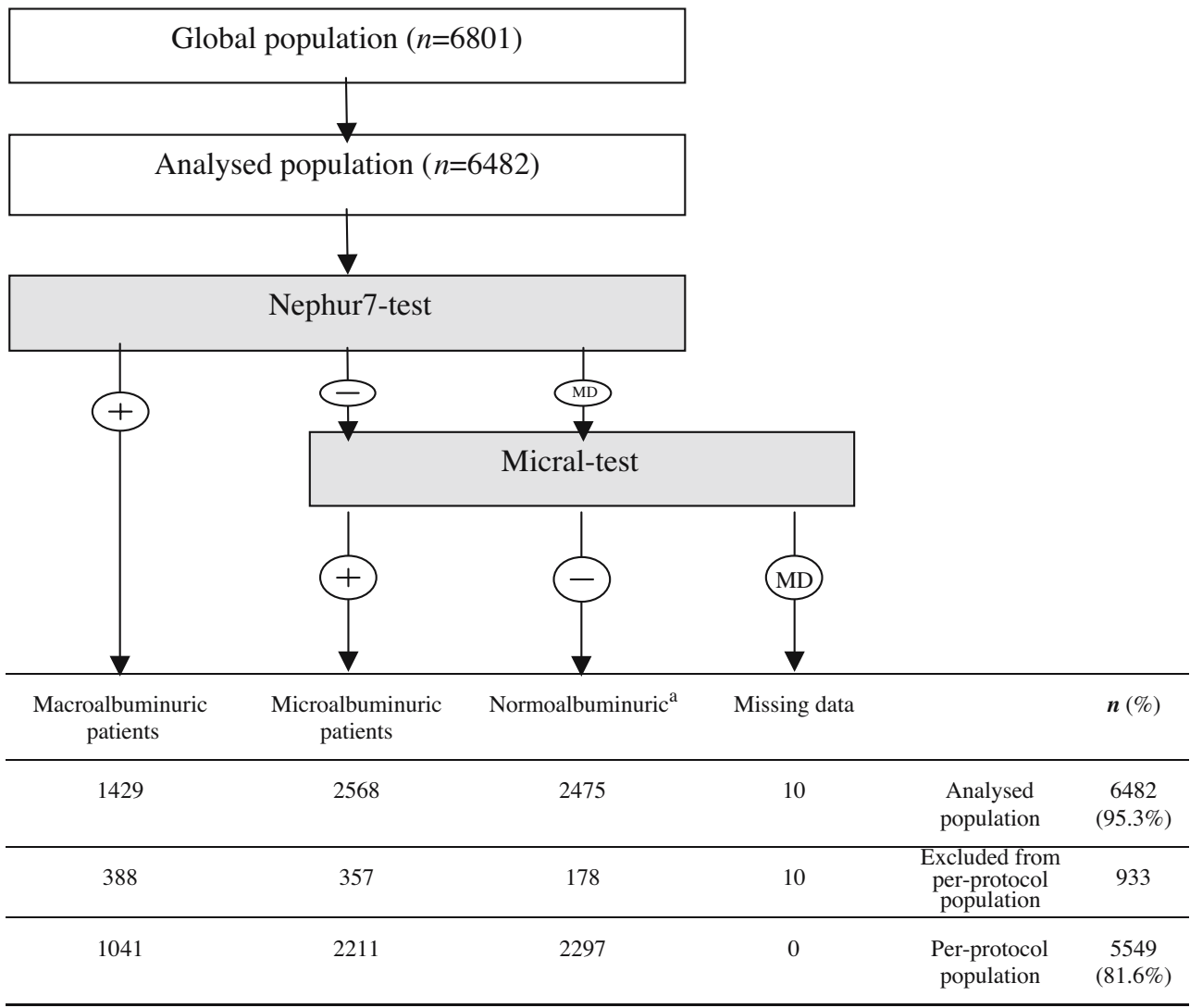

${ }^{\mathrm{a}}$ Patients with negative or missing albumin Nephur7-test and negative Micral-test population leaving the per-protocol population (Fig. 1). Further to the two-step screening, three albuminuric subgroups were defined: macroalbuminuric, microalbuminuric, normoalbuminuric.

Statistical analysis Quantitative variables were described by their means, standard deviations, and counts. Median and quartiles 1 and 3 were reported when appropriate. Qualitative variables were described by counts and percentages, missing data were included in the calculation of percentages. The three albuminuric subgroups were compared by one-way ANOVA for quantitative variables and by a chi square test for sex. The significance level was fixed at $5 \%$. Prevalence rates were calculated with a twosided 95\% CI. A two-step logistic regression analysis was performed on the per-protocol population. First, a univariate analysis determined the links between microalbuminuria (Yes/No) or macroalbuminuria (Yes/No) and the following 17 variables: ethnic subgroup, sex, age group, BMI, duration of diabetes classes, duration of hypertension classes, systolic blood pressure by class (high normal/ mild/moderate/severe/very severe) based on the 1999 WHO/ISH classification [15], diastolic blood pressure by class, cardiovascular complications (Yes/No), diabetic complications (Yes/No), smoking (Yes/No), diuretic intake (Yes/No), $\alpha$ blocker (Yes/No), $\beta$ blocker (Yes/No), calcium channel blocker (Yes/No), ACE inhibitor (Yes/No), and angiotensin II receptor blockers (ARB) intake (Yes/
No). A link between two variables was considered significant if the $p$ value test was 0.25 or less. Then, a stepwise logistic regression that only included the significant variables determined the best model to predict microalbuminuria or macroalbuminuria. All analyses were performed using SAS software version 8.02.

\section{Results}

Patient characteristics Patient characteristics of the perprotocol population $(n=5,549,81.6 \%$ of the enrolled population) are described in Table 1. No clinically significant difference in body weight and BMI was noted among the three subgroups. The duration of diabetes was significantly higher in the macroalbuminuric subgroup ( 8.0 years) than in the microalbuminuric (6.8 years) or normoalbuminuric (6.3 years) subgroups $(p<0.0001$; Table 2). The mean age at diagnosis of diabetes was similar (54.6 years) among the three subgroups. The mean $\mathrm{HbA}_{1} \mathrm{c}$ level was significantly higher in the macroalbuminuric subgroup $(8.2 \%$; documented results available in 541 patients, which corresponds to $52.0 \%$ of this population) than in the microalbuminuric subgroup $(7.9 \%$; $n=1,297,58.7 \%$ of microalbuminuria population) and the normoalbuminuric subgroup $(7.6 \% ; n=1,466,63.8 \%$ of normoalbuminuric population). Diabetic complications were particularly observed in macroalbuminuric patients 


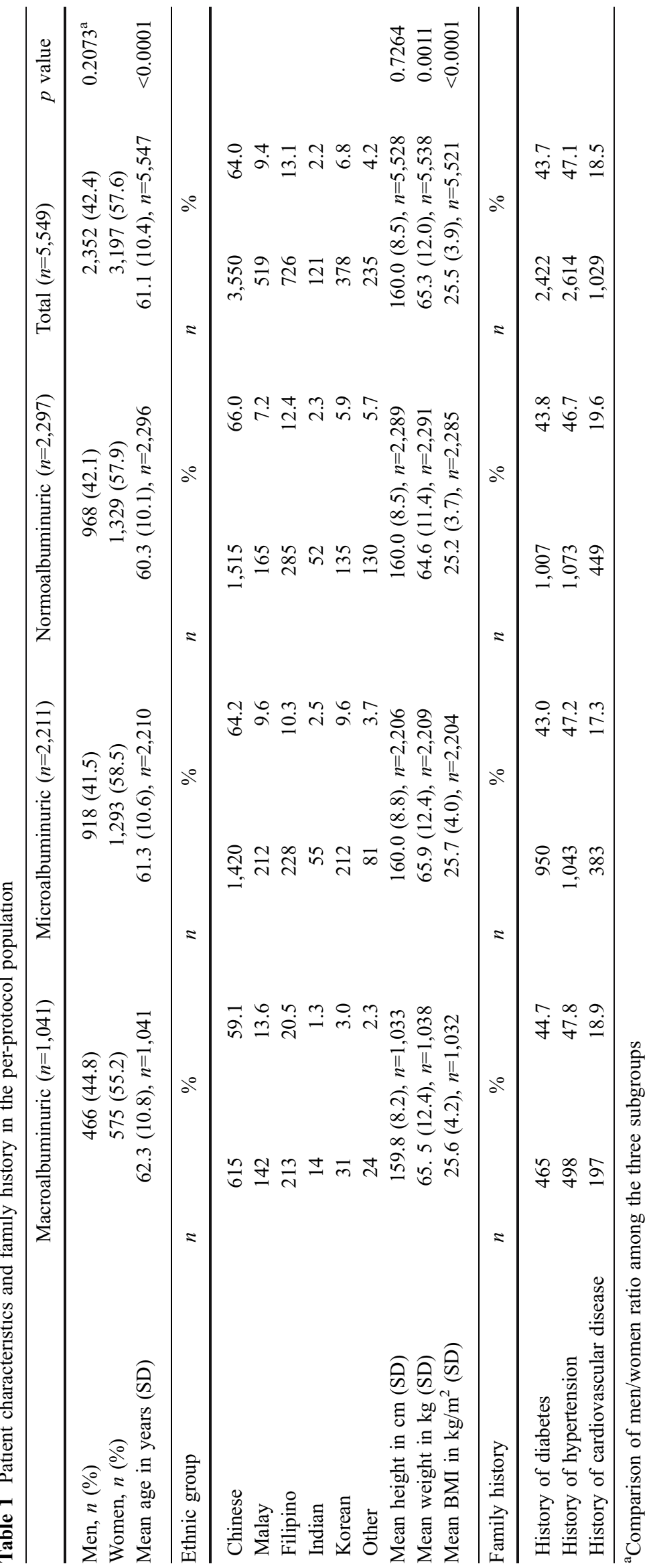


Table 2 Description of type 2 diabetes mellitus in the per-protocol population

\begin{tabular}{|c|c|c|c|c|c|c|c|c|c|}
\hline \multirow[b]{2}{*}{ Duration of diabetes (years) } & \multicolumn{2}{|c|}{$\begin{array}{l}\text { Macroalbuminuric } \\
(n=1,041)\end{array}$} & \multicolumn{2}{|c|}{$\begin{array}{l}\text { Microalbuminuric } \\
(n=2,211)\end{array}$} & \multicolumn{2}{|c|}{$\begin{array}{l}\text { Normoalbuminuric } \\
(n=2,297)\end{array}$} & \multicolumn{3}{|c|}{ Total $(n=5,549) p$ value } \\
\hline & & & & & & & & & \\
\hline Mean (SD) & \multicolumn{2}{|c|}{$8.0(7.1)$} & \multicolumn{2}{|c|}{$6.8(6.7)$} & \multicolumn{2}{|c|}{$6.3(6.1)$} & \multicolumn{2}{|c|}{$6.9(6.6)$} & $<0.0001$ \\
\hline Median & \multicolumn{2}{|c|}{6.0} & \multicolumn{2}{|c|}{5.0} & \multicolumn{2}{|c|}{5.0} & \multicolumn{2}{|l|}{5.0} & \\
\hline Q1 & \multicolumn{2}{|c|}{2.0} & \multicolumn{2}{|c|}{2.0} & \multicolumn{2}{|c|}{1.0} & \multicolumn{2}{|l|}{2.0} & \\
\hline \multirow[t]{2}{*}{ Q3 } & \multicolumn{2}{|c|}{12.0} & \multicolumn{2}{|c|}{10.0} & \multicolumn{2}{|c|}{10.0} & \multicolumn{2}{|l|}{10.0} & \\
\hline & $n$ & $\%$ & $n$ & $\%$ & $n$ & $\%$ & $n$ & $\%$ & \\
\hline$\leq 1$ year & 195 & 18.7 & 487 & 22.0 & 576 & 25.1 & 1,258 & 22.7 & \\
\hline$>1-5$ years & 274 & 26.3 & 704 & 31.8 & 724 & 31.5 & 1,702 & 30.7 & \\
\hline$>5-10$ years & 260 & 25.0 & 530 & 24.0 & 515 & 22.4 & 1,305 & 23.5 & \\
\hline$>10$ years & 312 & 30.0 & 490 & 22.2 & 482 & 21.0 & 1,284 & 23.1 & \\
\hline Known complications & $n$ & $\%$ & $n$ & $\%$ & $n$ & $\%$ & $n$ & $\%$ & \\
\hline At least 1 diabetic complication & 367 & 35.3 & 624 & 28.2 & 604 & 26.3 & 1,595 & 28.7 & \\
\hline Retinopathy & 213 & 20.5 & 323 & 14.6 & 321 & 14.0 & 857 & 15.4 & \\
\hline Peripheral neuropathy & 247 & 23.7 & 431 & 19.5 & 401 & 17.5 & 1,079 & 19.4 & \\
\hline Other neuropathy & 18 & 1.7 & 32 & 1.5 & 14 & 0.6 & 64 & 1.2 & \\
\hline Known cardiovascular complications & $n$ & $\%$ & $n$ & $\%$ & $n$ & $\%$ & $n$ & $\%$ & \\
\hline At least 1 cardiovascular complication & 306 & 29.4 & 542 & 24.5 & 535 & 23.3 & 1,383 & 24.9 & \\
\hline Previous stroke & 111 & 10.7 & 171 & 7.7 & 165 & 7.2 & 447 & 8.1 & \\
\hline Myocardial infarction & 45 & 4.3 & 68 & 3.1 & 58 & 2.5 & 171 & 3.1 & \\
\hline Congestive heart failure & 37 & 3.6 & 57 & 2.6 & 21 & 0.9 & 115 & 2.1 & \\
\hline $\begin{array}{l}\text { Mean fasting blood glucose in } \\
\mathrm{mmol} / \mathrm{l}(\mathrm{SD})\end{array}$ & 8.9 & ), $n=937$ & $8.5(3$ & $n=2,013$ & $8.1(2$ & $n=2,128$ & $\begin{array}{c}8.4 \\
n=5\end{array}$ & $\begin{array}{l}3.1), \\
078\end{array}$ & $<0.0001$ \\
\hline Mean $\mathrm{HbA}_{1} \mathrm{c}$ values in \% (SD) & 8.2( & ), $n=541$ & $7.9(1$ & $n=1,297$ & $7.6(1$ & $n=1,466$ & $\begin{array}{c}7.8 \\
n=3\end{array}$ & $\begin{array}{l}1.8) \\
304\end{array}$ & $<0.0001$ \\
\hline Mean serum creatinine in $\mu \mathrm{mol} / 1$ (SD) & 103.7 & .9), $n=707$ & $85.3(2$ & 9), $n=1,554$ & $83.4(2$ & 2), $n=1,723$ & $\begin{array}{r}87.7 \\
n=3\end{array}$ & $\begin{array}{l}32.5) \\
984\end{array}$ & $<0.0001$ \\
\hline
\end{tabular}

(35.3\%), while the percentage of complications observed in microalbuminuric patients was close to that in normoalbuminuric patients $(28.2 \%$ and $26.3 \%$, respectively; Table 2). Likewise, cardiovascular complications were more prevalent in macroalbuminuric patients (29.4\%), whereas percentages of cardiovascular complications in microalbuminuric and normoalbuminuric patients were similar (24.5\% and $23.3 \%$, respectively; Table 2$)$.

The mean duration of hypertension (8.5 years) was similar among the subgroups (Table 3 ). Mean systolic and diastolic blood pressure were significantly higher in macroalbuminuric patients $(148.3 / 85.0 \mathrm{~mm} \mathrm{Hg})$ than in microalbuminuric and normoalbuminuric patients $(142.9 / 83.3$ and $140.0 / 82.0 \mathrm{~mm} \mathrm{Hg}$, respectively). Only $11.6 \%$ of the patients had their systolic and diastolic blood pressure below the 130/80 $\mathrm{mm} \mathrm{Hg}$ target. The proportion of patients receiving an antihypertensive treatment $(89.0 \%)$ was homogeneous among the three subgroups, with $57.1 \%$ of them on monotherapy and $42.9 \%$ on combination therapy. Previously diagnosed lipid metabolism anomalies were observed in $48.8 \%$ of the patients, but less than $40 \%$ of them were receiving a lipid-lowering agent.
Primary endpoint The prevalence of microalbuminuria was $39.8 \%(39.2-40.5 ; 95 \% \mathrm{CI})$ and the prevalence of macroalbuminuria was $18.8 \%(18.2-19.3 ; 95 \% \mathrm{CI})$. The highest prevalence of microalbuminuria was observed in Korea (56.5\%) and the lowest in Pakistan (24.2\%) (Fig. 2). When analysed by type of practice, the highest prevalence of microalbuminuria was observed in patients attended by cardiology or nephrology specialists $(43.8 \%$ [41.8-45.7, $95 \% \mathrm{CI}]$ ) and the lowest in patients followed by general practitioners $(35.0 \%$ [33.6-36.4, 95\% CI]). For prevalence of macroalbuminuria, the opposite was observed, with the highest prevalence found in patients attending polyclinics/ hospital outpatient clinics $(26.9 \%[25.5-28.3,95 \% \mathrm{CI}])$ and the lowest in patients attended by cardiology or nephrology specialists $(13.2 \%$ [11.9-14.6, 95\% CI]).

In the multivariate analyses, the predictive factors for the presence of microalbuminuria were age greater than 59 years, elevated BMI, systolic blood pressure level greater than $139 \mathrm{~mm} \mathrm{Hg}$, and ethnic origin (Table 4). The statistically significant predictive factors for the presence of macroalbuminuria were age greater than 70 years, male sex, ethnic origin, elevated BMI, duration of diabetes 
Table 3 Description of hypertension in the per-protocol population

\begin{tabular}{|c|c|c|c|c|c|c|c|c|c|}
\hline \multirow[b]{2}{*}{ Duration of hypertension (years) } & \multicolumn{2}{|c|}{$\begin{array}{l}\text { Macroalbuminuric } \\
(n=1,041)\end{array}$} & \multicolumn{2}{|c|}{$\begin{array}{l}\text { Microalbuminuric } \\
(n=2,211)\end{array}$} & \multicolumn{2}{|c|}{$\begin{array}{l}\text { Normoalbuminuric } \\
(n=2,297)\end{array}$} & \multicolumn{2}{|c|}{ Total $(n=5,549)$} & \multirow[t]{2}{*}{$p$ value } \\
\hline & & & & & & & & & \\
\hline Mean (SD) & \multicolumn{2}{|c|}{$9.0(9.7)$} & \multicolumn{2}{|c|}{$8.7(9.5)$} & \multicolumn{2}{|c|}{$8.1(9.0)$} & \multicolumn{2}{|c|}{$8.5(9.3)$} & 0.0080 \\
\hline Median & \multicolumn{2}{|c|}{6.0} & \multicolumn{2}{|c|}{5.0} & \multicolumn{2}{|c|}{5.0} & \multicolumn{2}{|l|}{5.0} & \\
\hline Q1 & \multicolumn{2}{|c|}{2.0} & \multicolumn{2}{|c|}{2.0} & \multicolumn{2}{|c|}{2.0} & \multicolumn{2}{|l|}{2.0} & \\
\hline \multirow[t]{2}{*}{ Q3 } & \multicolumn{2}{|c|}{12.0} & \multicolumn{2}{|c|}{12.0} & \multicolumn{2}{|c|}{11.0} & \multicolumn{2}{|l|}{12.0} & \\
\hline & $n$ & $\%$ & $n$ & $\%$ & $n$ & $\%$ & $n$ & $\%$ & \\
\hline$\leq 1$ year & 211 & 20.3 & 503 & 22.8 & 521 & 22.7 & 1,235 & 22.3 & \\
\hline$>1-5$ years & 301 & 28.9 & 644 & 29.1 & 724 & 31.5 & 1,669 & 30.1 & \\
\hline$>5-10$ years & 222 & 21.3 & 419 & 19.0 & 465 & 20.2 & 1,106 & 19.9 & \\
\hline$>10$ years & 307 & 29.5 & 645 & 29.2 & 587 & 25.6 & 1,539 & 27.7 & \\
\hline $\begin{array}{l}\text { Mean systolic blood pressure in } \\
\mathrm{mm} \mathrm{Hg}(\mathrm{SD})\end{array}$ & \multicolumn{2}{|c|}{$148.3(21.0), n=1,040$} & \multicolumn{2}{|c|}{142.9 (17.9), $n=2,206$} & \multicolumn{2}{|c|}{140.0 (17.6), $n=2,292$} & \multicolumn{2}{|c|}{$\begin{array}{c}142.7(18.7) \\
n=5,538\end{array}$} & $<0.0001$ \\
\hline Systolic blood pressure by class & $n$ & $\%$ & $n$ & $\%$ & $n$ & $\%$ & $n$ & $\%$ & \\
\hline$\leq 130 \mathrm{~mm} \mathrm{Hg}$ (normal) & 224 & 21.5 & 640 & 29.0 & 809 & 35.2 & 1,673 & 30.2 & \\
\hline >130-139 (high normal) & 111 & 10.7 & 276 & 12.5 & 307 & 13.4 & 694 & 12.5 & \\
\hline$>139-159$ (mild) & 400 & 38.4 & 857 & 38.8 & 847 & 36.9 & 2,104 & 37.9 & \\
\hline$>159-179$ (moderate) & 220 & 21.1 & 341 & 15.4 & 270 & 11.8 & 831 & 14.5 & \\
\hline$>179-209$ (severe) & 72 & 6.9 & 90 & 4.1 & 55 & 2.4 & 217 & 3.9 & \\
\hline$>209$ (very severe) & 13 & 1.3 & 2 & 0.1 & 4 & 0.2 & 19 & 0.3 & \\
\hline $\begin{array}{l}\text { Mean diastolic blood pressure in } \\
\mathrm{mm} \mathrm{Hg}(\mathrm{SD})\end{array}$ & \multicolumn{2}{|c|}{$85.0(10.9), n=1,040$} & \multicolumn{2}{|c|}{$83.3(10.2), n=2,206$} & \multicolumn{2}{|c|}{$82.0(9.9), n=2,291$} & $\begin{array}{r}83.1 \\
n=5\end{array}$ & $\begin{array}{l}10.3), \\
537\end{array}$ & $<0.0001$ \\
\hline Diastolic blood pressure by class & $n$ & $\%$ & $n$ & $\%$ & $n$ & $\%$ & $n$ & $\%$ & \\
\hline$\leq 85$ mm Hg (normal) & 572 & 55.0 & 1,359 & 61.5 & 1,510 & 65.7 & 3,441 & 62.0 & \\
\hline$>85-89$ (high normal) & 45 & 4.3 & 139 & 6.3 & 146 & 6.4 & 330 & 6.0 & \\
\hline >89-99 (mild) & 300 & 28.8 & 528 & 23.9 & 489 & 21.3 & 1,317 & 23.7 & \\
\hline$>99-109$ (moderate) & 100 & 9.6 & 153 & 6.9 & 130 & 5.7 & 383 & 6.9 & \\
\hline$>109-119$ (severe) & 16 & 1.5 & 18 & 0.8 & 15 & 0.7 & 49 & 0.9 & \\
\hline$>119$ (very severe) & 7 & 0.7 & 9 & 0.4 & 1 & 0.0 & 17 & 0.3 & \\
\hline Systolic/diastolic blood pressure & $n$ & $\%$ & $n$ & $\%$ & $n$ & $\%$ & $n$ & $\%$ & \\
\hline$<130 / 80 \mathrm{~mm} \mathrm{Hg}$ & 85 & 8.2 & 234 & 10.6 & 326 & 14.2 & 645 & 11.6 & $<0.0001$ \\
\hline
\end{tabular}

longer than 5 years, presence of diabetic complications, diuretics intake, calcium channel blockers intake, diastolic and systolic blood pressure levels (Table 5).

\section{Discussion}

MAPS is the first large multicentre epidemiological study in Asia to determine the prevalence of microalbuminuria in type 2 diabetic patients with hypertension. This study indicates that $39.8 \%$ of the analysable population of 5,549 patients have microalbuminuria. This is higher than the prevalence rates, reported in population-based studies [16] for Western diabetic patients, which range from $17 \%$ to $21 \%$. In another Asian study, microalbuminuria was detected in $36.3 \%$ of type 2 diabetics at a diabetes centre in southern India [12]. The wide range in the prevalence of microalbuminuria in type 2 diabetics is probably due to genetic and cardiovascular risk factors (blood pressure, cholesterol, salt intake, etc.). In MAPS, most of the patients were Chinese (64\%). Compared to patients of Chinese origin, patients of Korean and Malay ethnic origin were most likely to have microalbuminuria. The high prevalence of microalbuminuria in non-Caucasians has also been observed in the Pima Indians of North America, where type 2 diabetes is very common, and more than $50 \%$ develop proteinuria within 20 years [17]. In type 2 diabetes, microalbuminuria is often associated with clustering of cardiovascular risk factors to form the metabolic syndrome [18]. High prevalence of metabolic syndrome has been reported in Asia [19, 20] with early onset of metabolic syndrome and diabetes mellitus in Asian Indians 


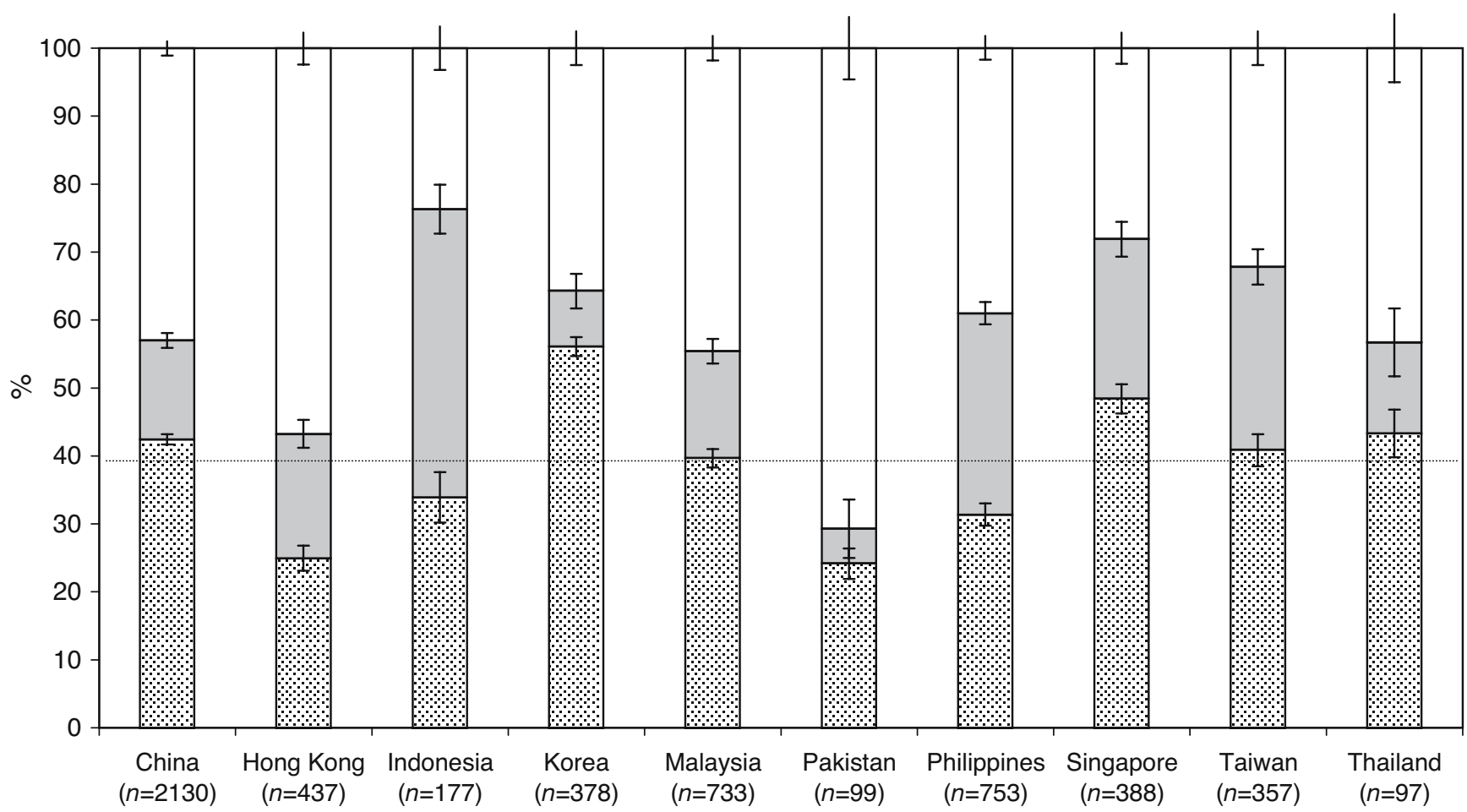

Fig. 2 Prevalence of microalbuminuria and macroalbuminuria (and $95 \%$ CI) by country in the per-protocol population. The dotted line represents the average prevalence of microalbuminuria $(39.8 \%)$.
Dotted bars indicate microalbuminuric; grey bars macroalbuminuric; white bars normoalbuminuric
$[19,21]$. In our study, over $48 \%$ of the patients had a known dyslipidaemia. Hypertriglyceridaemia, hypercholesterolaemia, high LDL cholesterol and low HDL cholesterol levels were reported among 58\%, 63\%, 42\%, and $23 \%$ of these patients, respectively. Drug therapy of dyslipidaemia was suboptimal as only $40 \%$ of patients were receiving a lipid-lowering agent.

Table 4 Predictive factors for the presence of microalbuminuria (odds ratio and $95 \% \mathrm{CI})^{\mathrm{a}}$

\begin{tabular}{lcc}
\hline Variables $(p \leq 0.05)$ & Odds ratio & Odds ratio CI \\
\hline Age $($ vs. $[30,49]$ years, $n=635)$ & & \\
$>59-70(n=1,766)$ & 1.216 & {$[1.005-1.472]$} \\
$>70(n=786)$ & 1.346 & {$[1.079-1.678]$} \\
BMI $\left(\mathrm{kg} / \mathrm{m}^{2}\right)$ & 1.042 & {$[1.026-1.059]$} \\
SBP levels (vs. normal, $n=1,449), \mathrm{mm} \mathrm{Hg}$ & \\
$>139-159(n=1,704)$ & 1.261 & {$[1.092-1.457]$} \\
$>159-179(n=611)$ & 1.619 & {$[1.330-1.969]$} \\
$>179-209(n=145)$ & 2.165 & {$[1.509-3.105]$} \\
Ethnic subgroup (vs. Chinese, $n=2,935)$ & \\
Korean $(n=347)$ & 1.761 & {$[1.396 ; 2.221]$} \\
Malay $(n=377)$ & 1.274 & {$[1.019 ; 1.593]$} \\
Other ${ }^{b}(n=209)$ & 0.613 & {$[0.455 ; 0.824]$} \\
\hline
\end{tabular}

$S B P$ systolic blood pressure

${ }^{\mathrm{a}}(n=4,508)$, per-protocol population less the macroalbuminuric patients

${ }^{\mathrm{b} U n c l a s s i f i e d ~ e t h n i c ~ s u b g r o u p ~(s e e ~ T a b l e ~ 1) ~}$
In addition to the $39.8 \%$ prevalence rate of microalbuminuria, $18.8 \%$ of 5,549 patients were found to have macroalbuminuria. As patients with previously known diabetic nephropathy or macroalbuminuria were excluded from screening, this high rate of macroalbuminuria was not anticipated and raises important questions about current screening strategies in diabetic patients.

There are a few limitations to the study results. Firstly, MAU detection was based on a single urine spot collection with semiquantitative dipstick determinations. The ADA guidelines [9] acknowledge that this technique has acceptable sensitivity and specificity, but recommend that positive tests be reconfirmed with more specific methods and, due to the marked day-to-day variability, that several collections be done in a 3- to 6-month period before designating a patient as having MAU. In this large crosssectional epidemiological study, the single urine collection and semiquantitative determination was judged adequate for the study objectives. Moreover, a within-trial validation of the Micral-test was performed by one of the authors (C.Y. Pan) in China. Micral-test results of 119 consecutive MAPS patients were compared with those obtained by immunochemical assay (DCA 2000+ commercial kit, Bayer Diagnostics, Germany) and 56 samples were compared with immunoturbidimetric determination (Beckman Array 360 system, USA). In comparison with DCA 2000+ (albumin/creatinine ratio), the Micral-test had an overall sensitivity of $91.9 \%$ and specificity of $63.4 \%$. In comparison with immunoturbidimetric assay, the overall sensitiv- 
Table 5 Predictive factors for the presence of macroalbuminuria (odds ratio and $95 \% \mathrm{CI})^{\mathrm{a}}$

$D B P$ diastolic blood pressure, $S B P$ systolic blood pressure a $(n=3,338)$, per-protocol population less the microalbuminuric patients

${ }^{b}$ Unclassified ethnic subgroup (see Table 1)

\begin{tabular}{|c|c|c|}
\hline Variables $(p \leq 0.05)$ & Odds ratio & Odds ratio $\mathrm{CI}$ \\
\hline Diuretic (Yes vs. No) $(n=580 / 2,758)$ & 1.319 & {$[1.073-1.621]$} \\
\hline Calcium channel blocker (Yes vs. No) $(n=1,221 / 2,117)$ & 1.332 & {$[1.126-1.575]$} \\
\hline \multicolumn{3}{|l|}{ DBP levels (vs. normal, $n=2,082$ ) } \\
\hline$>89-99 \mathrm{~mm} \mathrm{Hg}(n=1,317)$ & 1.474 & {$[1.198-1.813]$} \\
\hline$>119 \mathrm{~mm} \mathrm{Hg}(n=17)$ & 9.260 & [1.041-82.361] \\
\hline Sex (men vs. women) $(n=1,434 / 1,904)$ & 1.315 & {$[1.117-1.548]$} \\
\hline Diabetic complication (Yes vs. No) $(n=971 / 2,366)$ & 1.489 & {$[1.248-1.776]$} \\
\hline BMI $\left(\mathrm{kg} / \mathrm{m}^{2}\right)$ & 1.051 & {$[1.029-1.074]$} \\
\hline \multicolumn{3}{|l|}{ Age (vs. [30, 49] years, $n=451$ ) } \\
\hline$>70(n=628)$ & 2.063 & {$[1.519-2.801]$} \\
\hline \multicolumn{3}{|l|}{ Duration of diabetes subgroups (vs. $\leq 1$ year, $n=771$ ) } \\
\hline$>5-10$ years $(n=776)$ & 1.584 & {$[1.245-2.016]$} \\
\hline$>10$ years $(n=793)$ & 1.892 & {$[1.477-2.422]$} \\
\hline \multicolumn{3}{|l|}{ Ethnic subgroup (vs. Chinese, $n=2,130$ ) } \\
\hline Filipino $(n=498)$ & 2.256 & {$[1.800-2.829]$} \\
\hline Korean $(n=166)$ & 0.594 & {$[0.391-0.903]$} \\
\hline Malay $(n=307)$ & 1.873 & {$[1.435-2.446]$} \\
\hline Other $^{\mathrm{b}}(n=152)$ & 0.374 & {$[0.231-0.606]$} \\
\hline \multicolumn{3}{|l|}{ SBP levels (vs. normal, $n=1,033$ ) } \\
\hline$>130-139 \mathrm{~mm} \mathrm{Hg}(n=418)$ & 1.455 & {$[1.101-1.922]$} \\
\hline$>139-159 \mathrm{~mm} \mathrm{Hg}(n=1,247)$ & 1.599 & {$[1.295-1.975]$} \\
\hline$>159-179 \mathrm{~mm} \mathrm{Hg}(n=490)$ & 2.445 & {$[1.868-3.201]$} \\
\hline$>179-209 \mathrm{~mm} \mathrm{Hg}(n=127)$ & 3.708 & {$[2.388-5.757]$} \\
\hline$>209 \mathrm{~mm} \mathrm{Hg}(n=17)$ & 7.825 & {$[2.426-25.240]$} \\
\hline
\end{tabular}

ity and specificity of the Micral-test was $95 \%$ and $80 \%$, respectively.

Another possible limitation is that this was not a real population-based study. The extrapolation of these findings to the global type 2 diabetic and hypertensive population should be carefully evaluated. Nevertheless, at the study level, all efforts were made to have a balanced distribution between patients enrolled by diabetologists (47\%) and by non-diabetes specialists (53\%). And, at the country level, only representative physicians experienced in managing type 2 diabetic patients were selected and consecutive patients enrolled.

A third limitation concerns the blood test values as no systematic blood sampling was required per protocol. Only documented previous blood test results were recorded. For glycaemic control, $93.9 \%$ and $59.7 \%$ of patients had documented values of blood glucose and $\mathrm{HbA}_{1} \mathrm{c}$, respectively. For serum lipids, the available data rate varied between $72.8 \%$ (LDL cholesterol) and $81.6 \%$ of patients (triglycerides) with previously known dyslipidaemia. Importantly, the rates of available data were similar among the three subgroups.

Hypertension is common among diabetic patients and the prevalence is further increased in the presence of renal disease [22]. In MAPS, the mean blood pressure level was $143 / 83 \mathrm{~mm} \mathrm{Hg}$ and the mean duration of hypertension was 8.5 years. Systolic blood pressure was predictive of both microalbuminuria and macroalbuminuria. The presence of diabetic complications, duration of diabetes, and types of antihypertensive agent, i.e., diuretics and calcium channel blockers, were also predictive of macroalbuminuria. Although no conclusions could be drawn from a crosssectional study, these predictors are probably indicative of more severe underlying blood pressure elevation, and it is consistent with previous reports that dihydropyridine calcium channel blockers are not as effective as ACE inhibitors or ARB in alleviating albuminuria [23, 24]. Only $10.6 \%$ of patients with microalbuminuria and $8.2 \%$ with macroalbuminuria achieved blood pressure lower than 130/80 mm Hg. Eleven percent of our patients did not receive any antihypertensive therapy. The low control rates observed in this analysis reflect the prescribing habits of doctors in Asia, their perception of target blood pressure levels and the influence of guidelines in managing hypertension. These low control rates are not substantially different from many western countries [25]. As expected, severely elevated systolic blood pressure $(>179 \mathrm{~mm} \mathrm{Hg})$ was more common in the macroalbuminuric group. It is therefore important to develop strategies that increase the percentage of patients who achieve optimal blood pressure control as Asian type 2 diabetic patients have a higher risk of renal complications and stroke than their Caucasian counterparts [26].

Optimal blood pressure, tight glycaemic control and pharmacological blockade of the renin-angiotensin system with ACE inhibitors or ARB have been shown to decrease AER and decrease progression from incipient to overt nephropathy $[23,27]$. In the IRMA 2 study ("Irbesartan microalbuminuria type 2 diabetes mellitus in hypertensive patients"), hypertensive type 2 diabetic patients with 
microalbuminuria taking irbesartan $300 \mathrm{mg}$ daily had a significant $(70 \%, p<0.001)$ relative risk reduction for the development of diabetic nephropathy as measured by the changes in AER [28]. The RENAAL and IDNT trials have conclusively demonstrated the advantage of ARB therapy (losartan $100 \mathrm{mg}$ or irbesartan $300 \mathrm{mg}$ ) as part of a multidrug strategy to lower blood pressure and to prevent doubling of serum creatinine, end-stage renal disease or death in type 2 diabetic patients with hypertension and macroalbuminuria [23, 27]. There is compelling evidence that therapy that negatively modulates the renin-angiotensin system may be especially effective in reducing renal and cardiovascular disease in diabetic patients with hypertension. In the MAPS evaluation, ACE inhibitors and ARB were used in only $43.9 \%$ and $19.2 \%$ of patients, respectively. Less than $50 \%$ of patients were receiving two or more antihypertensive agents, even though a number of clinical trials have confirmed the need for multidrug therapies to reach target blood pressure in diabetes [29, 30].

Glycaemic control has been shown to prevent development of nephropathy and to reverse established pathology [31]. However, the majority of our patients in the microalbuminuric and macroalbuminuric groups did not achieve adequate glycaemic control as evidenced by available mean $\mathrm{HbA}_{1} \mathrm{c}$ values of over $7 \%$.

In conclusion, a $39.8 \%$ prevalence rate of microalbuminuria was observed in Asian type 2 diabetic and hypertensive patients. This high prevalence is alarming and indicates an impending pandemic of diabetic cardiovascular and renal disease in Asia with its potential economical burden. We strongly recommend implementing screening programmes for microalbuminuria in type 2 diabetic patients. Through cardiovascular risk reduction, lowering of blood pressure, renin-angiotensin system blockade and glycaemic control such early and targeted intervention could significantly reduce the occurrence of cardiovascular and renal complications.

Acknowledgements We would like to thank the 103 investigators and the monitoring teams of the ten participating countries and regions for their contribution to the study. This work was supported by a grant from Sanofi-Synthelabo.

\section{References}

1. Epstein M, Sowers JR (1992) Diabetes mellitus and hypertension. Hypertension 19:403-418

2. Stamler J, Vaccaro O, Neaton JD, Wentworth D (1993) Diabetic, other risk factors and 12 year cardiovascular mortality for men screened in Multiple Risk Factor Intervention Trial. Diabetes Care 16:434-444

3. Diabetes e-Atlas (2004) International Diabetes Foundation. Available from: http://www.idf.org/e-atlas/home/. Accessed 8 Jan 2004

4. World Health Organization (2004) The world health report 1997. Available from: http://www.who.int/whr2001/2001/archives/1997/index.htm. Accessed 8 Jan 2004

5. Dinneen SF, Gerstein HC (1997) The association of microalbuminuria in non-insulin-dependent diabetes mellitus. Arch Intern Med 157:1413-1418
6. Miettinen H, Haffner SM, Lehto S, Ronnemaa T, Pyorala K, Laakso M (1996) Proteinuria predicts stroke and other atherosclerotic vascular disease events in nondiabetic and non-insulin-dependent diabetic subjects. Stroke 27:2033-2039

7. Wang SL, Head J, Stevens L, Fuller JH (1996) Excess mortality and its relation to hypertension and proteinuria in diabetic patients. Diabetes Care 19:305-311

8. De Zeeuw D, Remuzzi G, Parving HH et al (2004) Proteinuria, a target for renoprotection in patients with type 2 diabetic nephropathy: lessons from RENAAL. Kidney Int 65:23092320

9. American Diabetes Association (2004) Nephropathy in diabetes. Position statement. Diabetes Care 27(Suppl 1):S79-S83

10. Spooren PF, Lekkerkerker JF, Vermes I (1992) Micral-test: a qualitative dipstick test for micro-albuminuria. Diabetes Res Clin Pract 18:83-87

11. Mather HM, Chaturvedi N, Kehely AM (1998) Comparison of prevalence and risk factors for microalbuminuria in South Asians and Europeans with type 2 diabetes mellitus. Diabet Med 15:672-677

12. Varghese A, Deepa R, Rema M, Mohan V (2001) Prevalence of microalbuminuria in type 2 diabetes mellitus at a diabetic center in South India. Post-Grad Med J 77:399-402

13. Tomura S, Kawada K, Saito K et al (1999) Prevalence of microalbuminuria and relationship to the risk of cardiovascular disease in the Japanese population. Am J Nephrol 19:13-20

14. Lee WRW, Lim HS, Thai AC et al (2001) A window in the current status of diabetes mellitus in Singapore-The DiabcareSingapore 1998 study. Singap Med J 42:501-507

15. Guidelines Sub-Committee (1999) World Health Organization - International Society of Hypertension guidelines for the management of hypertension. J Hypertens 17:151-183

16. Parving HH, Osterby R, Ritz E (2000) Diabetic nephropathy. In: BM Brenner (ed) The kidney. WB Saunders, Philadelphia, pp 1731-1773

17. Pettitt DJ, Saad MF, Bennett PH, Nelson RG, Knowler WC (1990) Familial predisposition to renal disease in two generations of Pima Indians with type 2 (non-insulin-dependent) diabetes mellitus. Diabetologia 33:438-443

18. Kuusisto J, Mykkanen L, Pyorala K, Laakso M (1995) Hyperinsulinemic microalbuminuria: a new risk indicator for coronary artery disease. Circulation 90:831-837

19. Ramachandran A, Snehalatha C, Vijay V (2004) Low risk threshold for acquired diabetogenic factors in Asian Indians. Diabetes Res Clin Pract 65:189-195

20. Oh JY, Hong YS, Sung YA, Barrett-Connor E (2004) Prevalence and factor analysis of metabolic syndrome in an urban Korean population. Diabetes Care 27:2027-2032

21. Misra A, Vikram NK (2004) Insulin resistance syndrome (metabolic syndrome) and obesity in Asian Indians: evidence and implications. Nutrition 20:482-491

22. Tarnow L, Rossing P, Gall MA, Nielsen FS, Parving HH (1994) Prevalence of arterial hypertension in diabetic patients before and after JNC-V. Diabetes Care 17:1247-1251

23. Lewis EJ, Hunsicker LG, Clarke WR et al (2001) Collaborative Study Group. Renoprotective effect of the angiotensin-receptor antagonist irbesartan in patients with nephropathy due to type 2 diabetes. N Engl J Med 345:851-860

24. Bakris GL, Weir MR, Secic M, Campbell B, Weis-McNulty A (2004) Differential effects of calcium antagonist subclasses on markers of nephropathy progression. Kidney Int 65:1991-2002

25. (1997) Sixth report of the joint national committee on prevention, detection, evaluation, and treatment of high blood pressure. Arch Intern Med 157:2413-2446

26. Morrish NJ, Wang SL, Stevens LK, Fuller JH, Keen H (2001) Mortality and causes of death in the WHO multinational study of vascular disease in diabetics. Diabetologia 44(Supp1 2):S14 S21

27. Parving $\mathrm{HH}$, Lehnert $\mathrm{H}$, Brochner-Mortensen J, Gomis R, Andersen S, Arner P (2001) The effect of irbesartan on the development of diabetic nephropathy in patients with type 2 diabetes. N Engl J Med 345:870-878 
28. Brenner BM, Cooper ME, De Zeeuw D et al (2001) Effects of losartan on renal and cardiovascular outcomes in patients with type 2 diabetes and nephropathy. N Engl J Med 345:861-869

29. Bakris GL, Williams M, Dworkin L et al (2000) Preserving renal function in adults with hypertension and diabetes: a consensus approach. National Kidney Foundation Hypertension and Diabetes Executive Committees Working Group. Am J Kidney Dis 36:646-661
30. The JNC-7 Report (2003) The 7th report of the joint national committee on prevention, detection, evaluation, and treatment of high blood pressure. JAMA 289:2560-2572

31. UKPDS 33 (1998) Intensive blood glucose control with sulphonylurea or insulin compared with conventional treatment and risk of complications in NIDDM. Lancet 352:837-852 